\title{
NONLINEAR MANY-STAGED DIFFUSION
}

\author{
A.J. Janavičíus
}

Šiauliai University, P. Višinskio 25,5400 Šiauliai, Lithuania

(Received November 25, 1997; revised version April 20, 1998)

We considered and solved the nonlinear diffusion equation formerly. The more complicated but more useful task of many-staged diffusion is solved in this paper. The obtained solution satisfies the initial distribution of the impurities and can be generalized for many-staged diffusion. Using these solutions we can take into account all the stages of a planary transistor formation.

PACS numbers: $66.30 .-\mathrm{h}$

\section{Introduction}

In Ref. [1] the following nonlinear diffusion equation was proposed:

$$
\frac{\mathrm{d}}{\mathrm{d} t} N=\frac{\mathrm{d}}{\mathrm{d} x}\left(D \frac{\mathrm{d}}{\mathrm{d} x} N\right) \text {, }
$$

where the diffusion coefficient was directly proportional to the impurities concentration

$$
D(t, N)=D_{\mathrm{n} 1} N(x, t), \quad D_{\mathrm{n} 1}=\frac{D_{0}}{N_{\mathrm{a}}} \mathrm{e}^{-E / k T_{1}},
$$

where $D_{0}$ is pre-exponential factor and $E$ is the activation * nergy of the diffusion in solids. The concentration dependence of the diffusion coefficient $D$ was defined by $N_{\mathrm{a}}[1,2]$.

The similarity solution of the nonlinear diffusion equation was obtained in a form of the power series of similarity variable $\xi_{1}$. We will use the approximate solution [2]

$$
N\left(x, t_{1}\right)_{1}=N_{\mathrm{s} 1} f\left(\xi_{1}\right)_{1}, \quad \xi_{1}=\frac{x}{\sqrt{D_{1} t_{1}}},
$$

$$
\begin{aligned}
& f\left(\xi_{1}\right)_{1}=\left[1-0.4439 \xi_{1}-0.09855\left(\xi_{1}\right)^{2}-6.754 \times 10^{-3}\left(\xi_{1}\right)^{3}+3.582 \times 10^{-4}\left(\xi_{1}\right)^{4}\right] \\
& D_{1}=D_{\mathrm{n} 1} N_{\mathrm{s} 1}, N_{\mathrm{a}}=N_{\mathrm{s} 1}, 0 \leq \xi_{1} \leq \xi_{01}, \xi_{01}=1.615, x_{01}=1.615 \sqrt{D_{1} t_{1}}
\end{aligned}
$$


which for the first-stage diffusion from infinity source satisfies the following boundary:

$$
N(0, t)_{1}=N_{s 1}, \quad 0 \leq t \leq t_{1}, \quad N\left(x \geq x_{01}, t_{1}\right)_{1}=0
$$

and initial

$$
N(x, 0)_{1}=0, \quad x>0
$$

conditions. In this paper we will solve the important drive-in or second stage diffusion task, which is useful for the base formation of the bipolar transistor.

\section{Mathematical model of the many-staged diffusion of impurities atoms}

Nonlinear equation of diffusion (1) is being considered for the second stage diffusion with the following boundary conditions:

$$
\begin{aligned}
& \lim _{t \rightarrow \infty}\left(\frac{\mathrm{d}}{\mathrm{d} x} N(x, t)\right)_{x=0}=0, \\
& \lim _{x \rightarrow \infty} N\left(x \geq x_{0}, t\right)=0, \quad t=\mathrm{const}
\end{aligned}
$$

and initial condition

$$
N(x, 0)=N\left(\boldsymbol{x}, \boldsymbol{t}_{1}\right)_{1} \text {. }
$$

We can solve the drive-in problem going to a new similarity variable

$$
\xi(x, t)=\frac{x}{\left(D_{1} t_{1}\right)^{1 / 6}\left(D_{1} t_{1}+D_{2} t\right)^{1 / 3}}, \quad D_{2}=D_{\mathrm{n} 2} N_{\mathrm{s} 1},
$$

where $D_{2}$ is the diffusion coefficient for the second diffusion stage. The solution of diffusion equation (1) in this case has the form

$$
N(\xi)=N_{\mathrm{s} 1} F(t) f(\xi), \quad N(0,0)=N_{\mathrm{s} 1}, \quad F(t)=\frac{\left(D_{1} t_{1}\right)^{1 / 3}}{\left(D_{1} t_{1}+D_{2} t\right)^{1 / 3}} .
$$

Here $D_{1}$ and $D_{2}$ are diffusion coefficients, while $t_{1}$ and $t$ are diffusion times for first and second diffusion stages, respectively. tion:

Substituting (10), (9), (2) into (1) we obtain the following differential equa-

$$
\frac{\mathrm{d}}{\mathrm{d} \xi}\left(f(\xi) \frac{\mathrm{d}}{\mathrm{d} \xi} f(\xi)\right)+\frac{1}{3} \frac{\mathrm{d}}{\mathrm{d} \xi} \xi f=0 .
$$

Taking into account initial conditions (8) and (10) we can obtain the initial condition for $f(\xi)$

$$
\lim _{\xi \rightarrow \xi_{1}} f(\xi)=f\left(\xi_{1}\right)_{1}
$$

After integrating the last equation from $\xi_{1}$ to $\xi$ we get

$$
\frac{\mathrm{d}}{\mathrm{d} \xi} f(\xi)^{2}+\frac{1}{3} \xi f(\xi)=\frac{\mathrm{d}}{\mathrm{d} \xi_{1}} f\left(\xi_{1}\right)^{2}+\frac{1}{3} \xi_{1} f\left(\xi_{1}\right) .
$$

From this equation and initial condition (12) we obtain the very useful relation

$$
f(\xi)=f\left(\xi_{1}\right)_{1}, \quad \xi=\xi_{1} .
$$


Considering the initial conditions for (9), (10) and the last expression, we decide that the function $f(\xi)$ describing the drive-in diffusion has the same expression as the function $f\left(\xi_{1}\right)_{1}$ describing the introduction or first stage diffusion (3), only the latter depends on other similarity variable $\xi$. Then solution (10) of Eq. (1) satisfying boundary (6), (7) and initial (8) conditions can be presented in the following form:

$$
N(x, t)=N_{\mathrm{s} 1} F(t) f(\xi)_{1} .
$$

The number of the introduced impurities atoms in the first diffusion stage

$$
Q_{1}=\int_{0}^{x_{01}} N(x, t)_{1} \mathrm{~d} x
$$

can be obtained from (3)

$$
Q_{1}=0.5492 N_{\mathrm{s} 1} x_{01} \text {. }
$$

For the second stage from (10), (9) we obtained the same result.

From (3), (15), (9) we obtain the maximum penetration of the impurities after the drive-in diffusion for the time $t_{i}$ :

$$
x_{0 i}=1.615\left(D_{1} t_{1}\right)^{1 / 6}\left(D_{1} t_{1}+D_{2} t_{i}\right)^{1 / 3} .
$$

From formulas (3), (17) and (18) we obtain very useful relation between surface concentrations and penetration depths

$$
N_{\mathrm{s} 1} x_{01}=N_{\mathrm{s} i} x_{0 i}, \quad N_{\mathrm{s} i} x_{0 i}=1.82 Q_{1}, \quad N_{\mathrm{s} i}=N_{\mathrm{s} 1} F\left(t_{i}\right), \quad i=2,3,4 \ldots
$$

Substituting (9) into (3) and using (10) we obtain

$$
\left(\frac{\mathrm{d}}{\mathrm{d} x} N\right)_{x=0}=-0.4439 N_{\mathrm{s} 1} \frac{\left(D_{1} t_{1}\right)^{1 / 6}}{\left(D_{1} t_{1}+D_{2} t\right)^{2 / 3}} .
$$

From the last expression we can see that the first derivative of impurities concentration at the origin tends to zero while the time of second stage of diffusion tends to infinity because drive-in of impurities from the surface occurs very slowly. The high initial concentration of the impurities at the surface generates the decreasing current density

$$
j=-D_{0}\left(N \frac{\mathrm{d}}{\mathrm{d} x} N\right)_{x=0}
$$

which we can obtain from formulas (15), (21)

$$
j(t)=0.4439 D_{2} N_{\mathrm{s} 1} \frac{\left(D_{1} t_{1}\right)^{1 / 2}}{D_{1} t_{1}+D_{2} t} .
$$

From (22) we obtain the expression

$$
\frac{j(t)}{j(0)}=\frac{D_{1} t_{1}}{D_{1} t_{1}+D_{2} t}
$$

for the ratio of the current density to the current density at the initial moment. This ratio tends to zero when $D_{2} t \gg D_{1} t_{1}$. 


\section{Conclusions}

In Refs. $[3,4]$ the dependence of diffusion coefficients on the impurity concentration near the surface was considered. For high concentration arsenic or boron diffusion in silicon we can count up that diffusion coefficients are directly proportional to this concentration. For high phosphorus concentration [4] the situation is more complicated. But even in this case [1] the theoretically calculated profile fits very closely to the experiment while the fitting of the classical solution $N_{\text {erf }}$ to the experimental profile tail region is impossible [1,2].

When the first stage diffusion contains the diffusion from the infinity source and following $n$ stages drive-in diffusion, we must substitute in (9), (10), (15):

$$
D_{1} t_{1}+D_{2} t
$$

with

$$
D_{1} t_{1}+D_{2} t_{2}+D_{3} t_{3}+\ldots+D_{n} t .
$$

The many-staged diffusion boron in silicon with the first stage obtained by the ion implantation $[5,6]$ is compared with the theoretical normalized profiles $X, Z, Y$ calculated by (3), (9), (10), (15) in Fig. 1. The ion implanted profile $X$ localized at the surface can be approximately described by (3) with $D_{1} t_{1}=3.834 \times 10^{-3} \mu \mathrm{m}^{2}$. This approximation can be obtained from the implantation depth $0.1 \mu \mathrm{m}$ and (3). When the drive-in time is long enough, the difference between the real profile of implanted impurities and first stage profile of thermal diffusion does not play any role for definition of drive-in profiles. The drive-in profiles $Z$ and $Y$ were calculated at diffusion coefficient $D=10^{-6} \mu \mathrm{m}^{2} / \mathrm{s}$ and



Fig. 1. Ion implanted boron in silicon profile $X_{i}-$, drive-in profiles $Z\left(t_{2}\right) \ldots$ - $Y\left(t_{3}\right)---$ at $t_{2}=60 \mathrm{~min}, t_{3}=240 \mathrm{~min}$ and $D=10^{-6} \mu \mathrm{m}^{2} / \mathrm{s}$. 
times $t_{2}=60 \mathrm{~min}, t_{3}=240 \mathrm{~min}$ consequently. The obtained normalized profiles satisfy the relation (19) with high accuracy. There we obtain $x_{02}=0.168 \mu \mathrm{m}$, $N_{\mathrm{s} 2} / N_{\mathrm{s} 1}=0.802, x_{03}=0.228 \mu \mathrm{m}, N_{\mathrm{s} 3} / N_{\mathrm{s} 1}=0.595, x_{02} N_{\mathrm{s} 2} / N_{\mathrm{s} 1}=0.135 \mu \mathrm{m}$, $x_{03} N_{\mathrm{s} 3} / N_{\mathrm{s} 1}=0.136 \mu \mathrm{m}$. The obtained results and Eqs. (17), (19) show that conservation of an amount of the implanted ions of boron is also satisfied with sufficient accuracy. The many-staged model presented in this paper is more general and includes the first stage diffusion [2] as initial condition. For long-time second-stage diffusion when $D_{2} t>D_{1} t_{1}$ we obtain that penetration depth (18) $x_{0}$ is proportional to $t^{1 / 3}$ and drive-in diffusion occurs more slowly than in the case of first stage (3). The same time dependence was obtained for the frontier of the nonlinear diffusion [7] from finite impurities source in the zero point. In this case $N_{\mathrm{si}} x_{0 i}=1.5 Q_{1}$. The penetration depths $x_{2}=0.17 \mu \mathrm{m}, x_{3}=0.23 \mu \mathrm{m}$ and $N_{2} / N_{1}=0.65, N_{3} / N_{1}=0.4$ presented in $[5,6]$ are in good accordance for depths and worse for surface concentrations obtained above. The last difference is the result of borons diffusion into $\mathrm{SiO}_{2}$ and an exact conservation of impurities for profiles in $[5,6]$ is not satisfied. It is remarkable that the obtained solutions (3), (10), (15) include the same function $f$ which depends on different similarity variitlles (3), (9) defined in the same region. It means that solutions for all diffusion stages are found with the same accuracy. The similarity variables method proposed in this paper is more advanced than Laplace's transformations method [8] for solving the diffusion drive-in task. Using the obtained solutions (3), (10), (15) we can take into account all the diffusion stages of a planar transistor formation.

\section{References}

[1] A.J. Janavičius, V. Stukaitè, D.J. Zanevičius, Electronics Technique, Ser. 2, Semicond. Dev., Issue 1 (160), 27 (1983) (in Russian).

[2] A.J. Janavičius, Phys. Lett. A 224, 159 (1997).

[3] W.-S. Wang, Y.-H. Lo, IEEE Trans. Electron Devices ED-30, 1828 (1983).

[4] A.N. Bubenikov, Modeling Integral Microtechnologies, Devices and Schemes, High School, Moskva, 1989, p. 320 (in Russian).

[5] D.S. Perloff, J. Electrochem. Soc. 120, 1135 (1973).

[6] H. Ryssel, I. Ruge, Ion Implantation, Science, Moskva 1983, p. 360 (in Russian).

[7] Y.F. Kozlov, A.I. Sidorov, Electronics Technique, Ser. 6, Materials, Issue 5 (130), 71 (1979) (in Russian).

[8] P.J. Gedgaudas, A.J. Janavičius, Electronics T'echnique, Ser. 3, Microelectronics, Issue 4 (120), 66 (1986) (in Russian). 